\title{
Coupling of long-wavelength density fluctuations to orientations in cellulose nanocrystal suspensions under external fields
}

\author{
Kyongok Kang $\odot^{*}$ \\ Forschungszentrum Jülich, Institute of Complex Systems, ICS-3, D-52425 Jülich, Germany \\ Pascal Bertsch and Peter Fischer ${ }^{\dagger}$ \\ Institute of Food Nutrition and Health, ETH Zurich, 8092 Zurich, Switzerland
}

(Received 20 May 2019; revised manuscript received 12 August 2019; published 14 November 2019)

\begin{abstract}
Motivated by the development of cellulose-based functional materials, we investigate the microscopic dynamics of suspensions of cellulose nanocrystals $(\mathrm{CNCs})$ at different ionic strengths, both in the absence and in the presence of $\mathrm{AC}$ electric fields and for various temperatures. A concentration of $5 \mathrm{wt} \%$ of the CNCs is chosen for which the dispersions are in the full chiral-nematic state at low ionic strengths. Dynamic light scattering is used to characterize the wave vector-dependent decay rates of number-density fluctuations. Contrary to an isotropic suspension, the dispersion relations (the wave vector dependence of the correlation-function decay rates) as obtained by means of depolarized light scattering are found to exhibit anomalous behavior. The dispersion relations, both without and with an external field, exhibit minima at small wave vectors, which is attributed to coupling of translational motion to the orientation of the CNCs, shown in the chiral-nematic state. The location of the minima is found to weakly depend on ionic strength and shifts significantly towards larger wave vectors upon applying an external electric field for sufficiently high ionic strengths. Finally, preliminary results are presented for smaller length-scale density fluctuations (at larger wave vectors) as a function of temperature, revealing the anisotropic mobilities in the chiral-nematic state of CNCs.
\end{abstract}

DOI: 10.1103/PhysRevE.100.052606

\section{INTRODUCTION}

Various applications of cellulose have been developed, such as alternative ecofriendly thermal insulator materials which consist of aligned nanocellulose fibers [1] and the ion-induced formation of hydrogels and glasses consisting of nematically ordered cellulose nanocrystals [2] using both monovalent and divalent salts [3]. There are, however, still challenges to overcome in the further development of other types of cellulose-based functional materials, which may replace currently used synthetic materials.

The anisotropic shape of cellulose nanocrystals [commonly abbreviated as (CNCs)] gives rise to a chiral-nematic phase at sufficiently low ionic strengths where in the optical pitch is reported to depend on the ionic strength $[4,5]$. The length of individual cellulose nanocrystals is on the order of a few hundreds of nanometers, resulting in chiral-nematic domains with sizes of a few microns. In the present paper, we study the dynamics of CNC suspensions with a concentration of 5 wt $\%$. At low ionic strengths, upto about $5 \mathrm{mM}$ added

\author{
*k.kang@fz-juelich.de \\ †pascal.bertsch@hest.ethz.ch, peter.fischer@ hest.ethz.ch
}

Published by the American Physical Society under the terms of the Creative Commons Attribution 4.0 International license. Further distribution of this work must maintain attribution to the author(s) and the published article's title, journal citation, and DOI.
$\mathrm{NaCl}$, such suspensions are in the full chiral-nematic state. On further increasing the ionic strength to about $10 \mathrm{mM}$ added salt, the chiral-nematic state becomes unstable, and a gel-like solid suspension is obtained. Due to the diminished range of the electrostatic interactions for these high ionic strengths, the attractive forces between the CNCs come into play, leading to gelation. Aggregation of the CNCs occurs for ionic strengths larger than about $20 \mathrm{mM}$ [6].

External fields couple to the particle dynamics and affect microstructural order of particles through their surface charges, electric dipole moment, as well as the electrical double layer in the case of electric fields, and to their magnetic dipole moment in the case of magnetic fields. Suspensions of very long and thin highly charged colloidal rods (fd-virus particles), for example, exhibit various phase transitions and dynamical states depending on the electric-field amplitude and frequency [7]. Spherical colloidal particles with a magnetic dipole moment are known to form chainlike structures under the influence of an external magnetic field, exhibiting two distinct dynamical modes as observed by means of dynamic light scattering [8,9]. Field-induced (chiral)-nematic alignment and phase transitions in CNC suspensions, in dependence of the ionic strength and $p \mathrm{H}$, have been observed by means of scanning electron microscopy [2] and rheology [10]. Field-induced alignment of silicon-oil-based cellulose suspensions by means of electric fields has been discussed in Ref. [11].

What has so far not been resolved is the underlying particle dynamics both in the absence and in the presence of electric 
(a)
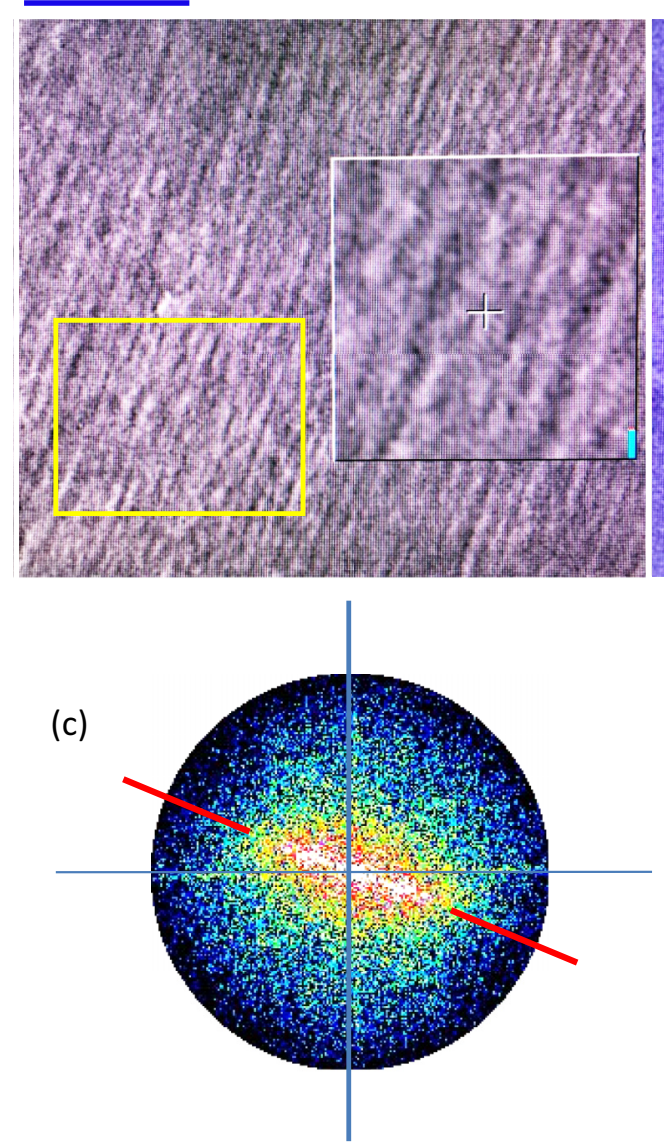

(b)

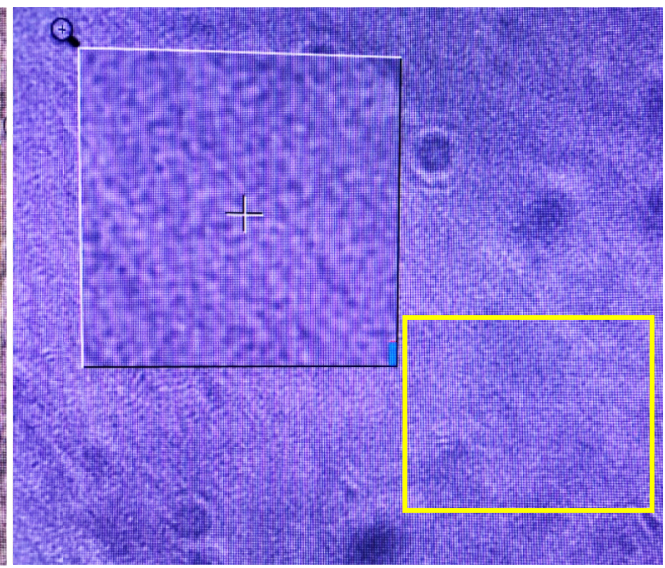

(d)

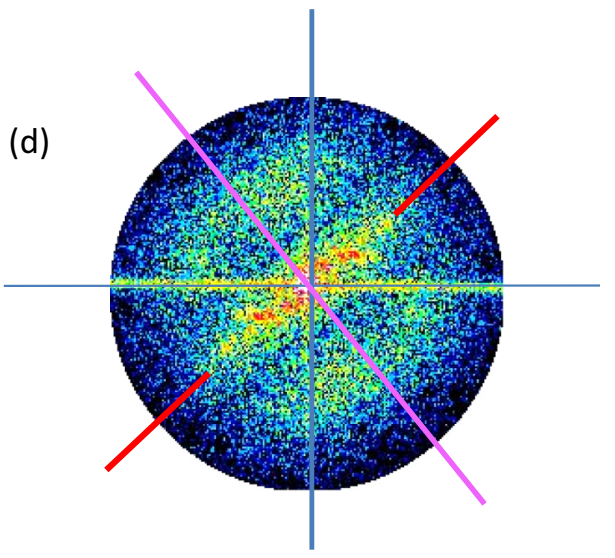

FIG. 1. Depolarized optical images of the morphologies of homogenized CNC suspensions of 5 wt $\%$ at a low electric field amplitude of $25 \mathrm{~V} / \mathrm{mm}$ and a frequency of $100 \mathrm{~Hz}$ at (a) $0 \mathrm{mM}$ and (b) $2 \mathrm{mM} \mathrm{NaCl}$ salt concentration. The scale bar in (a) corresponds $150 \mu \mathrm{m}$. The corresponding Fourier transforms (FTs) are given in (c) and (d). With $2 \mathrm{mM}$ added salt, the chiral-nematic state is further enhanced as a stable state, shown evidently by the two additional lobes in the FT in (d). The estimated Fourier spacing is $2 \pi / 100 \mu \mathrm{m}$.

fields. The aim of the present paper is, thus, to characterize the length-scale (or wave vector-) dependent diffusion properties of concentrated CNC suspensions (as obtained from natural renewable sources of celluloses) at different $\mathrm{NaCl}$ salt concentrations and temperatures by means of light scattering with and without an external electric field. A fundamental understanding of the dynamical behavior of cellulose nanocrystals may ultimately serve to develop a rational design of processing parameters of cellulose-based materials.

\section{MATERIALS AND EXPERIMENTAL METHODS}

CNCs were provided by Celluforce (Montreal, Canada), which have an average length of $79 \pm 6 \mathrm{~nm}$ and a thickness of $5 \mathrm{~nm}$ with a polydispersity in length of about $50 \%$ and with an effective linear charge density of $0.67 e \mathrm{~nm}^{-1}$ (with $e<0$ as the elementary charge). The CNCs are dispersed in Milli-Q water (Milliporesigma) with the addition of an amount of $1 \mathrm{M}$ solution of $\mathrm{NaCl}$ (Thermo Fischer) to establish the required ionic strength. The suspensions are then sonicated with a Hielscher UP200S to eliminate CNC aggregation. The CNC suspensions are not homogeneous without sonication. Sonication is required to fully deagglomerate the CNCs and obtain single crystallites $[12,13]$. However, in contrast to the longer more amorphous cellulose nanofibrils, sonication does not result in a further size reduction. Once dispersed, the CNCs form thermodynamically stable suspensions and do not aggregate due to electrostatic repulsion at sufficiently low ionic strength (lower than about $20 \mathrm{mM}$ added $\mathrm{NaCl}$ ) [6]. Photographs of CNC suspensions can be found in Ref. [2]. Figure 1 shows depolarized optical morphologies of homogenized CNC suspensions, in the presence of an AC electric field with an amplitude of $25 \mathrm{~V} / \mathrm{mm}$ and a frequency of $100 \mathrm{~Hz}$ for (a) $0 \mathrm{mM}$ (no added salt) and (b) $2 \mathrm{mM} \mathrm{NaCl}$ where the chiral-nematic states are stable (see the discussion in the Introduction). The corresponding FTs of the depolarized images are shown in Figs. 1(c) and 1(d). The two lobes in the FT in Fig. 1(d) originate from the chiral-nematic pitch. These lobes, however, are not visible in Fig. 1(c). The reason for this is that the chiral-nematic pitch is now larger to such an extent that the two lobes merge with the central part of the FT. The larger pitch at lower ionic strength is the result of the longer ranged repulsive electrostatic interactions, which more effectively screen the chiral core-core interactions that are responsible for the existence of the chiral-nematic state [4,5].

Two independent homemade dynamic light scattering (DLS) setups are used to probe density fluctuations: (i) One is a standard wide-angle DLS, which probes scattering wave 

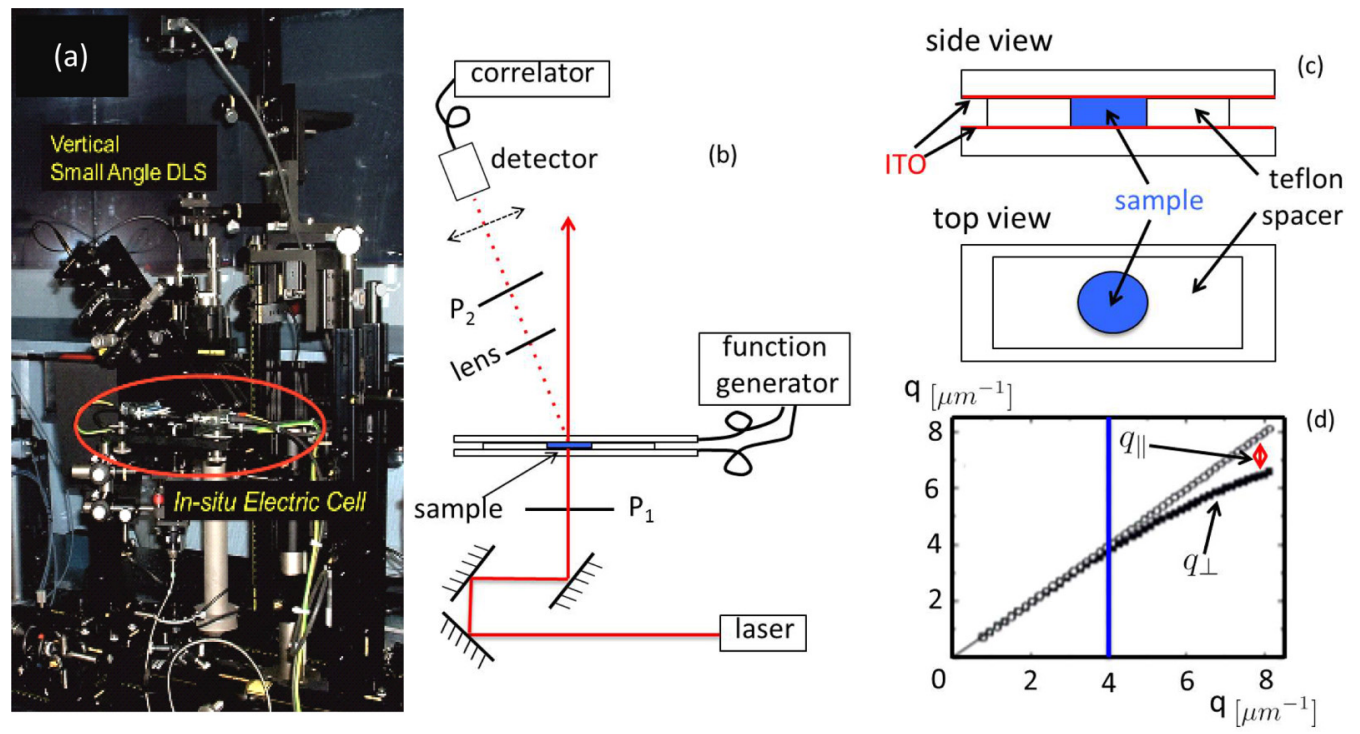

FIG. 2. (a) A real image view and (b) a simple schematic of the small-angle SAeDLS setup. Three mirrors are used to be able to adjust the position and direction of the laser beam. The sample is contained between two flat glass plates, that are coated with conducting indium tin oxide (ITO) layers which are connected to a function generator. The detector resides on a goniometer with its center of rotation at the position of the sample and is connected to a correlator. The two polarizers $P_{1}$ and $P_{2}$ are used to switch between vertically polarized incident light (VV) and horizontally polarized detected light ( $\mathrm{VH})$ modes. A lens is used that focuses in the sample in between the two plates. (c) A side and top view of the electric sample cell. The conducting ITO layers are in contact with the sample, which is contained within a circular niche in a teflon spacer. (d) The wave vector develops a component parallel to the vertical direction for wave vectors beyond $q=4 \mu \mathrm{m}^{-1}$.

vectors in the range of $q \sim 9-30 \mu \mathrm{m}^{-1}$, equipped with a $21 \mathrm{~mW}$ JDS Uniphase 1145P laser with a wavelength of $632.8 \mathrm{~nm}$ and with a cylindrical cuvette of $5 \mathrm{~mm}$ diameter. The scattered light is collected by means of a fiber, the output of which is connected to an ALV SO-SIPD photomultiplier assembly (PMT). The digital output of the PMT is processed by an ALV "FastCorr correlator" with a linear sampling-time spacing (which can be adjusted from $75 \mathrm{~ns}$ to $400 \mathrm{~s}$ ). The scattering cell is immersed in a refractive index matching silicon oil bath to avoid heterodyning and to control the temperature. It takes typically about $60 \mathrm{~min}$ to obtain a reliable correlation function. (ii) The other DLS setup is a small-angle electricfield dynamic light scattering (SAeDLS) instrument, an image of which is given in Fig. 2(a) and a schematic in Fig. 2(b). This setup probes much smaller scattering wave vectors in the range of $q \sim 1.8-4.8 \mu \mathrm{m}^{-1}$ and allows to apply an electric field. Three mirrors are used to be able to adjust the location and direction of the incident laser beam. Two polarizers are used to adjust the polarization directions of the incident and scattered light. With the use of a lens (with a focal length of $75 \mathrm{~mm}$ ), which focuses in the middle of the gap between the two flat electrodes, away from the electrode surfaces, scattering contributions from particles close to the walls of the electrical cell are eliminated. The scattering experiments, thus, probe the dynamics of CNCs in bulk without contributions from CNC-wall interactions. A schematic of the sample cell is given in Fig. 2(c). This in situ electric cell consists of two flat ITO coated glasses (from Präzisions Glas und Optik $\mathrm{GmbH}, \mathrm{CEC} 500 \mathrm{~S}$ ) with dimensions $40 \times 70 \mathrm{~mm}^{2}$ and with a thickness of $0.7 \mathrm{~mm}$. The ITO layer has a high visible light transmission $(\sim 90 \%$ at $633 \mathrm{~nm}$, and the coating thickness is $\sim 15 \mathrm{~nm}$. The amount of sample is $300 \mu \mathrm{l}$ that is loaded on the bottom plate within a niche of insulating polytetrafluoroethy- lene (PTFE) (teflon) film spacer (Armbrecht and Matthes GmbH, AR5038 and AR5038GP), and the sample thickness is $1.0 \mathrm{~mm}$. The upper electrode is then gently placed onto the lower plate. The two plates are then sealed with the same PTFE tape in order to avoid evaporation and to fixate the two electrodes. The ITO coatings are located on the side of the sample. All measurements are performed at the center of the sample to ensure a homogeneous electric field. The ITO layers are then connected to a function generator (Avtech model AV-151G-B, $1 \mathrm{~Hz}-350 \mathrm{kHz}$, maximum $\pm 200 \mathrm{~V}$, load resistance $50 \mathrm{k} \Omega$ ) by means of electronic connection pins. A sinusoidally varying $\mathrm{AC}$ electric potential is applied to the electrodes. A more detailed description of the setup is provided in Ref. [14]. The vertical SAeDLS setup can, thus, be used to probe the microscopic dynamics of CNCs under the influence of an external electric field. The field amplitudes in the present paper are in the range of $25-50 \mathrm{~V} / \mathrm{mm}$, and the frequency is fixed to $100 \mathrm{~Hz}$. The SAeDLS setup probes fluctuations in directions parallel to the electrodes for wave vectors less than about $4 \mu \mathrm{m}^{-1}$. For larger scattering wave vectors, also density fluctuations are probed in directions perpendicular to the electrodes along the direction of the incident laser beam as can be seen from the plot in Fig. 2(d). In the presence of an electric field, which aligns the CNCs along the field direction, the SAeDLS setup, thus, probes translational motion perpendicular to the direction of alignment for wave vectors less than $4 \mu \mathrm{m}^{-1}$. Due to scattering by the glass-water and glass-air interfaces, there is typically about $20 \%$ heterodyning that contributes to the measured intensity correlation function. It takes about 60 min to measure a single correlation function.

Correlation functions are measured in the $\mathrm{VH}$ and $\mathrm{VV}$ modes where the first $\mathrm{V}$ refers to vertically polarized incident light and the second $\mathrm{V}$ or $\mathrm{H}$ refers to vertically or horizontally 
polarized detected light, respectively. Contrary to the VV mode, the VH mode is susceptible to the orientation of the cellulose particles as will be discussed below. The wide-angle DLS setup in used in both VV and VH modes and the SAeDLS in the VH mode.

\section{THE INTERPRETATION OF VV- AND VH-SCATTERING CORRELATION FUNCTIONS}

Expressions for the intensity correlation functions for anisotropic particles include time-dependent single-particle scattering amplitudes which are a function of the instantaneous orientation of the particles (details can be found in, for example, Ref. [15]). For rodlike particles for which the thickness is much smaller than the inverse scattering vector, such as the CNCs, the scattering amplitude is equal to $\sin \{L \mathbf{q}$. $\hat{\mathbf{u}}(t) / 2\} /[L \mathbf{q} \cdot \hat{\mathbf{u}}(t) / 2]$ with $L$ as the length of the CNC and $\hat{\mathbf{u}}$ as the unit vector along the long axis of the CNC. For the SAeDLS setup, the maximum variation of the scattering amplitude is from 1 for $\mathbf{q} \perp \hat{\mathbf{u}}$ to 0.96 for $\mathbf{q} \| \hat{\mathbf{u}}$, whereas for the wide-angle DLS setup, it varies from 1 to 0.87 . Rotational motion, therefore, leads to minor changes in the scattered intensity and can, therefore, be neglected in the interpretation of correlation functions.

For VV-mode scattering from homogeneous equilibrium suspensions of rodlike colloids with a sufficiently small depolarization ratio, such as the $\mathrm{CNCs}$, the scattered-field autocorrelation function $g_{1}$ is given by (apart from a time-independent prefactor) [15],

$$
\begin{aligned}
g_{1}^{\mathrm{VV}} & \sim \frac{1}{N}\left\langle\sum_{i, j=1}^{N} \exp \left\{i \mathbf{q} \cdot\left[\mathbf{r}_{i}(0)-\mathbf{r}_{j}(t)\right]\right\}\right\rangle \\
& =\frac{1}{N} \sum_{i, j=1}^{N} \int d \mathbf{r}_{i} \int d \mathbf{r}_{j} P\left(\mathbf{r}_{i}, \mathbf{r}_{j} \mid t\right) \exp \left\{i \mathbf{q} \cdot\left(\mathbf{r}_{i}-\mathbf{r}_{j}\right)\right\},
\end{aligned}
$$

where $\mathbf{q}$ is the scattering vector, $N$ is the number of CNCs within the scattering volume, and where $\mathbf{r}_{i}(t)$ is the position coordinate of CNC number $i$ at time $t$. The brackets $\langle\cdots\rangle$ denote averaging with respect positions, whose average is explicitly written in the second line in Eq. (1) with $P\left(\mathbf{r}_{i}, \mathbf{r}_{j} \mid t\right)$ as the probability density function (pdf) for the position of $\mathrm{CNC} i$ at time 0 and of CNC $j$ and time $t$.

Under the above discussed neglect of orientational motion of the CNCs, the scattered-field autocorrelation function in the $\mathrm{VH}$ mode is given by [15]

$$
\begin{aligned}
g_{1}^{\mathrm{VH}} \sim & \frac{1}{N}\left\langle\sum_{i, j=1}^{N}\left[\hat{\mathbf{n}}_{s} \cdot \hat{\mathbf{u}}_{i}(0) \hat{\mathbf{u}}_{i}(0) \cdot \hat{\mathbf{n}}_{0}\right]\left[\hat{\mathbf{n}}_{s} \cdot \hat{\mathbf{u}}_{j}(t) \hat{\mathbf{u}}_{j}(t) \cdot \hat{\mathbf{n}}_{0}\right]\right. \\
& \left.\times \exp \left\{i \mathbf{q} \cdot\left[\mathbf{r}_{i}(0)-\mathbf{r}_{j}(t)\right]\right\}\right\rangle \\
= & \frac{1}{N} \sum_{i, j=1}^{N} \oint d \hat{\mathbf{u}}_{i} \oint d \hat{\mathbf{u}}_{j} P\left(\hat{\mathbf{u}}_{i}, \hat{\mathbf{u}}_{j} \mid t\right)\left[\hat{\mathbf{n}}_{s} \cdot \hat{\mathbf{u}}_{i} \hat{\mathbf{u}}_{i} \cdot \hat{\mathbf{n}}_{0}\right] \\
& \times\left[\hat{\mathbf{n}}_{s} \cdot \hat{\mathbf{u}}_{j} \hat{\mathbf{u}}_{j} \cdot \hat{\mathbf{n}}_{0}\right] \int d \mathbf{r}_{i} \int d \mathbf{r}_{j} P_{c}\left(\mathbf{r}_{i}, \mathbf{r}_{j}\left|\hat{\mathbf{u}}_{i}, \hat{\mathbf{u}}_{j}\right| t\right) \\
& \times \exp \left\{i \mathbf{q} \cdot\left(\mathbf{r}_{i}-\mathbf{r}_{j}\right)\right\}
\end{aligned}
$$

where $\hat{\mathbf{n}}_{s}$ and $\hat{\mathbf{n}}_{0}$ are the perpendicular polarization directions of the scattered and incident light, respectively, and where $\hat{\mathbf{u}}_{i}(t)$ is the unit vector along the long axis of CNC number $i$ at time $t$. The integrals $\oint$ range over all orientations, that is, over the unit spherical surface. The brackets $\langle\cdots\rangle$ now include averaging with respect to orientations. In the second equation in (2), $P_{c}\left(\mathbf{r}_{i}, \mathbf{r}_{j}\left|\hat{\mathbf{u}}_{i}, \hat{\mathbf{u}}_{j}\right| t\right)$ is the conditional pdf for the position $\mathbf{r}_{i}$ at time 0 and $\mathbf{r}_{j}$ at time $t$, given the prescribed orientations $\hat{\mathbf{u}}_{i}$ at time 0 and $\hat{\mathbf{u}}_{j}$ at time $t$, whereas $P\left(\hat{\mathbf{u}}_{i}, \hat{\mathbf{u}}_{j} \mid t\right)$ is the (unconditional) pdf of $\hat{\mathbf{u}}_{i}$ and $\hat{\mathbf{u}}_{j}$ at time 0 and $t$, respectively. Since the conditional pdf $P_{c}\left(\mathbf{r}_{i}, \mathbf{r}_{j}\left|\hat{\mathbf{u}}_{i}, \hat{\mathbf{u}}_{j}\right| t\right)$ in Eq. (2) is a sensitive function of CNC orientation at the high concentrations under consideration, $\mathrm{VH}$ experiments probe the orientation dependence of the relaxation of density waves (with wavelengths $2 \pi / q$ ). Rotational diffusion is strongly hindered so that the pdf $P\left(\hat{\mathbf{u}}_{i}, \hat{\mathbf{u}}_{j} \mid t\right)$ varies relatively slow with time. The relaxation of the VH-correlation functions is, therefore, essentially determined by the time dependence of $P_{c}\left(\mathbf{r}_{i}, \mathbf{r}_{j}\left|\hat{\mathbf{u}}_{i}, \hat{\mathbf{u}}_{j}\right| t\right)$. In comparison to VV experiments, there will be an additional slow mode that signifies those orientations for which density waves relax relatively slowly. We will refer to this mode as the rotational-coupling mode.

To summarize, correlation functions obtained in the VV mode probe the relaxation of density waves with a wavelength $2 \pi / q$ corresponding to the time dependence of the unconditional pdf $P\left(\mathbf{r}_{i}, \mathbf{r}_{j} \mid t\right)$, whereas the relaxation of density waves as probed in the $\mathrm{VH}$ mode corresponds to the time dependence of the conditional pdf $P_{c}\left(\mathbf{r}_{i}, \mathbf{r}_{j}\left|\hat{\mathbf{u}}_{i}, \hat{\mathbf{u}}_{j}\right| t\right)$, which couples the positional probability to the orientations of the CNCs.

Within the chiral-nematic state, the VV-correlation functions are, therefore, expected to exhibit two relaxation modes, one related to translational motion along the director, and a slower mode for translational motion perpendicular to the director. The simplest form of the field-correlation function is, therefore,

$$
\hat{g}_{1}^{\mathrm{VV}}(t)=A_{\|} \exp \left\{-\Gamma_{\|} t\right\}+A_{\perp} \exp \left\{-\Gamma_{\perp} t\right\}
$$

where $A_{\|}$and $A_{\perp}=1-A_{\|}$are the amplitudes for the fast and slow modes related to translational diffusion parallel and perpendicular to the chiral-nematic director, respectively. The corresponding relaxation rates are denoted by $\Gamma_{\|}$and $\Gamma_{\perp}$. Due to the above mentioned coupling to rotation, the $\mathrm{VH}$ correlation function exhibits three relaxation modes,

$$
\begin{aligned}
\hat{g}_{1}^{\mathrm{VH}}(t)= & A_{\|} \exp \left\{-\Gamma_{\|} t\right\}+A_{\perp} \exp \left\{-\Gamma_{\perp} t\right\} \\
& +A_{r} \exp \left\{-\left(\Gamma_{r} t\right)^{\beta}\right\},
\end{aligned}
$$

where $A_{r}=1-A_{\|}-A_{\perp}$ is the amplitude of the slowest mode related to the adjustment of orientational order during relaxation of density waves. Here, a stretching exponent $\beta$ is included to account for the polydispersity in length for which the rotational mode is most sensitive (the length polydispersity in the CNC samples used in the present paper is 50\%).

In a homodyne scattering experiment (with the wideangle DLS setup), the relation between the normalized fieldcorrelation function $\hat{g}_{1}$ and the experimentally measured intensity correlation function $\tilde{g}_{2}$ is given by the Siegert relation $\tilde{g}_{2}=I^{2}\left[1+C \hat{g}_{1}^{2}\right]$, where $I$ is the average scattered intensity and $C$ is the dynamical contrast which is close to unity. Experimental results for correlation functions are given in 
terms of the normalized correlation function $g_{2}=\left(\tilde{g}_{2} / I^{2}\right)-$ 1 , which varies from a value close to one for $t=0$ to zero for long times. In a heterodyne scattering experiment (with the SAeDLS setup), the extended Siegert relation reads $\tilde{g}_{2}=$ $A_{0}+A_{1} \hat{g}_{1}+A_{2} \hat{g}_{1}^{2}$ where the amplitudes $A_{0-2}$ depend on the ratio of the scattered light as compared to the light that is mixed in due to scattering from the glass interfaces of the scattering cell. The intercept at $t=0$ of heterodyne correlation functions is significantly smaller than those for homodyne correlation functions. For graphical clarity, experimental results for heterodyne correlation functions are given in terms of a normalized correlation function $g_{2}$ that varies from 1 to 0 . Such a normalization does not affect relaxation rates. The fitting function in Eq. (4) together with the homodyne Siegert relation will be shown to accurately describe the experimentally obtained homodyne $\mathrm{VH}$-correlation functions. Anomalous dispersion relations are found, however, at very small scattering angles, which are probed with the SAeDLS setup which operates in heterodyne mode: At the very small angles probed with this setup, heterodyning is unavoidable, especially with the scattering cell that enables to apply an external electric field. The fitting function in Eq. (4) together with the generalized heterodyne Siegert relation now involves ten fitting parameters [the two independent amplitudes in Eq. (4), three relaxation rates, the stretching exponential, the three constants $A_{0-2}$ is the generalized Siegert relation, and a small base line]. It turns out that these many fitting parameters results in quite large errors in the relaxation rates. The heterodyne correlation functions can, therefore, not be fitted to Eqs. (3) and (4). We will, therefore, use the following procedure to characterize the relaxation rate for translational diffusion. As will be seen in Sec. IV C where homodyne VHcorrelation functions as obtained with the wide-angle DLS setup are fitted to Eq. (4), and the amplitudes of the fast and intermediate modes (motion parallel and perpendicular to the director, respectively) are quite small: They add up to about $10 \%$ of the decay of $g_{2}$. For all the experimental results obtained here, the major decay is, thus, due to the slow rotational-coupling mode discussed above. The corresponding translational relaxation rate can, therefore, be characterized by the reciprocal time at which the normalized intensity correlation function relaxed to, say, 0.5 or 0.7 . Furthermore, the change in the relaxation rate is as large as a few decades, which is much larger than the factor of 2 that would be present due to heterodyning (the terms linear and quadratic in $\hat{g}_{1}$ in the generalized Siegert relation). In comparing decay rates for the small wave vectors as obtained with the SAeDLS setup and those for the larger wave vectors as obtained with the wide-angle DLS setup, the same procedure to obtain decay rates will be used for the latter.

\section{RESULTS AND DISCUSSION}

\section{A. Density fluctuations of CNCs without added salt}

For a CNC suspension with a concentration of $5 \mathrm{wt} \%$ without added salt, plots of the measured normalized intensity autocorrelation functions for various values of the wave vector as obtained from the SAeDLS setup in the VH mode and for the wide-angle DLS setup in the VH and VV modes are given in Figs. 3(a)-3(c), respectively. The arrows in these plots indicate increasing scattering wave vectors. As discussed in the previous section, the characteristic relaxation rate is determined from the inverse time at which the correlation functions decayed to 0.5 (as will be seen later, the typical wave vector dependence does not change when this value is chosen to be 0.7 instead of 0.5 ). The resulting decay rates are given in Fig. 3(d). Fits of the correlation functions in Fig. 3(a) with the heterodyne Siegert relation and the fitting function (4) is possible but with large errors in the decay rates, suggesting the same anomalous wave vector dependence for the slow translational mode as given in Fig. 3(d) for the small wave vectors. The black data points in Fig. 3(d) are the decay rates in the VV mode, which exhibit the expected $\sim q^{2}$ dependence (see the inset in this figure). That the data points at the very low wave vectors are below the $q^{2}$ line in the inset is due to the quite large size polydipersity of the CNCs. The solid black line in Fig. 3(d) in the main part of the figure is the same $q^{2}$ line as in the inset. The translational diffusion coefficient that corresponds to the slope of the $q^{2}$ line is $0.06 \mu \mathrm{m}^{2} / \mathrm{s}$. This value for the diffusion coefficient is comparable to other source types of cellulose materials: The translational diffusion coefficient for cotton is equal to $0.06 \mu \mathrm{m}^{2} / \mathrm{s}$, and that for wood is $0.01 \mu \mathrm{m}^{2} / \mathrm{s}$ for a similar concentration of $5.5 \mathrm{wt} \%$ [16]. The red data points are the decay rates obtained from the wide-angle DLS experiments in the VH mode, and the blue data points from the SAeDLS setup in the $\mathrm{VH}$ mode. The coupling of translational fluctuations to CNC orientation (as discussed in the previous section) clearly leads to a severe slowing down of their relaxation with two distinct minima (the solid line is a guide to the eye). Since light scattering probes density fluctuations with a wavelength equal to $2 \pi / q$, the occurrence of minima in the dispersion relation indicates the existence of particle orientations for which relaxation of density fluctuations is relatively slow for particular welldefined wavelengths. A microscopic understanding of this phenomenon requires extensive simulations on large systems, which is beyond the scope of the present paper. Dynamical slowing down can have a structural origin where the minima in decay rates correspond to maxima in the structure factor, which is commonly referred to as de Gennes narrowing. Since the scattering amplitude of the CNCs hardly changes within the $q$ range under consideration, the scattered intensity should exhibit maxima in case de Gennes narrowing occurs. Figure 3(e) shows intensity time traces from which correlation functions are obtained, and Fig. 3(f) shows that the scattered intensity as a function of the wave vector. The latter clearly reveals that structural maxima are not present so that de Gennes narrowing is not responsible for the minima in the observed dispersion relation in Fig. 3(d).

Figure 4(a) shows the depolarized VH-intensity autocorrelation functions in the low- $q$ regime in the presence of an AC electric field with an amplitude of $50 \mathrm{~V} / \mathrm{mm}$ and with a frequency of $100 \mathrm{~Hz}$. For reasons that will become clear later, the characteristic translational decay time is now determined as the reciprocal time at which the normalized correlation function decayed to 0.7 (instead of 0.5). Figure 4(b) shows the corresponding dispersion relation in the absence of the electric field [the blue data points are obtained from the correlation functions given in Fig. 3(a)] and with the electric field (the 

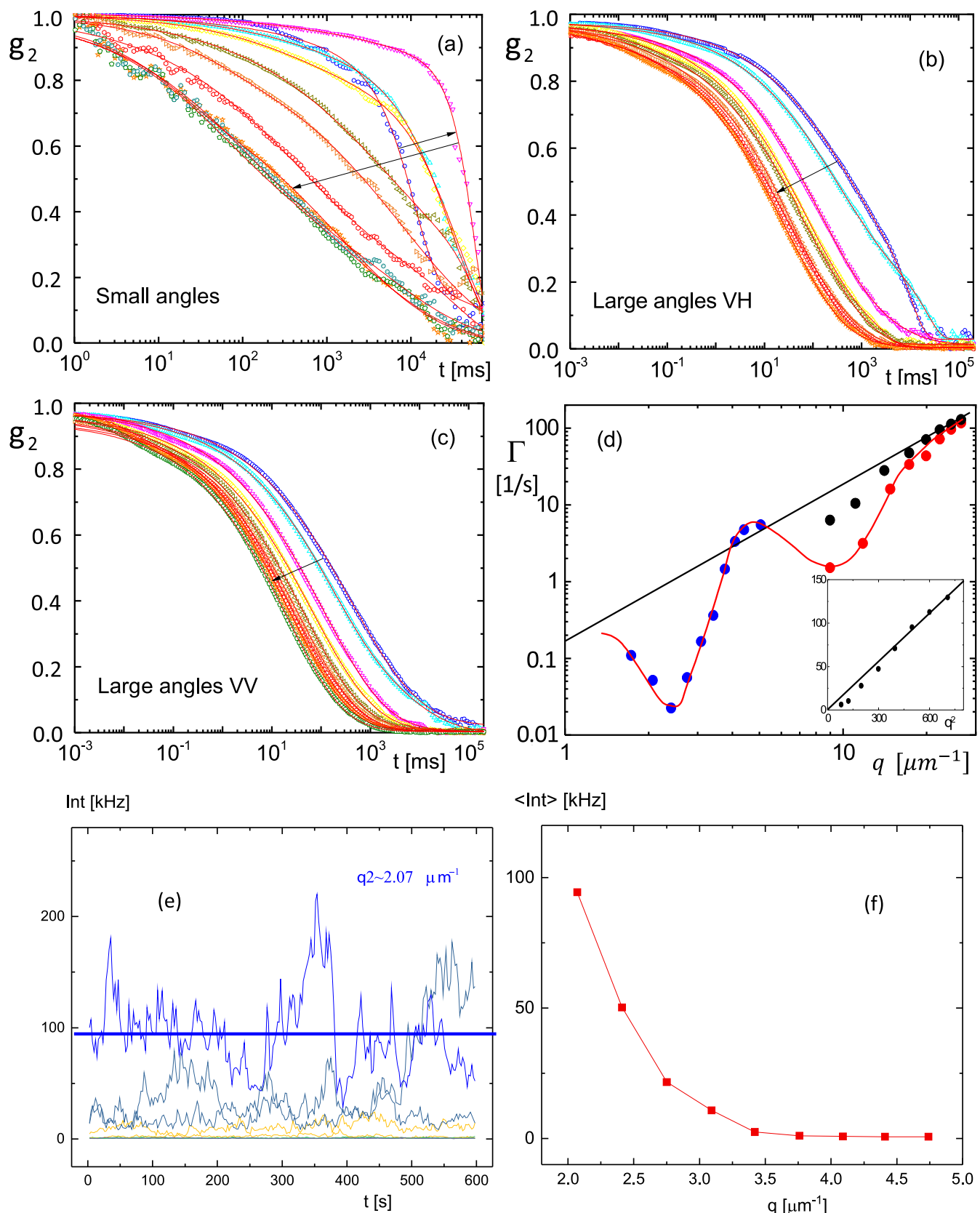

$<\operatorname{lnt}>[\mathrm{kHz}]$

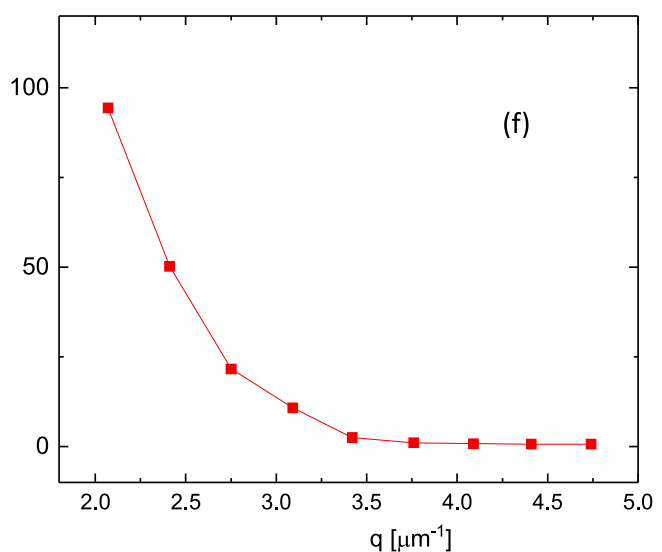

FIG. 3. (a) Normalized intensity autocorrelation functions for small wave vectors in the VH mode as obtained with the SAeDLS setup, and (b) and (c) correlation functions at larger wave vectors as obtained with the wide-angle DLS setup in the VH mode and VV mode, respectively. The corresponding dispersion relations are shown in (d): Blue and red data points are for the $\mathrm{VH}$ mode, and the black points are for the VV mode. The decay rates are determined as the reciprocal time at which the normalized correlation functions decayed to a value of 0.5 . The solid black line in (d) corresponds to an $\sim q^{2}$ dependence, which is also shown in the inset where the decay rate is plotted on a linear scale against $q^{2}$. The arrows in (a)-(c) indicate increasing wave vector $q$. The red solid line in (d) is a guide to the eye. The color code is as follows: (a) (dark blue, $q=1.73 \mu \mathrm{m}^{-1}$ ), (light blue, 2.07), (magenta, 2.41), (yellow, 2.75), (light green, 3.09), (orange, 3.42), (red, 3.76), (orange stars, 4.09), (dark green, 4.41), and (b) and (c) (dark blue, $q=8.98 \mu \mathrm{m}^{-1}$ ), (light blue, 11.8), (magenta, 14.8), (yellow, 17.3), (light green, 19.9), (orange stars, 22.3), (red, 24.5), (orange, 26.6). (e) Examples of intensity time traces for the SAeDLS small-angle setup from which correlation functions are calculated and (f) the averaged scattered intensity as a function of the wave vector.

red data points). As can be seen from the blue data points and solid line (a guide to the eye), the same dispersion relation with a distinct minimum at small wave vectors is found as in Fig. 3(d) where the decay value is 0.5 . Note that the first data point for the decay rates in Fig. 4(b) with and without field coincide. Furthermore, the dispersion relation at the low ionic strength under consideration, without added salt, is slightly shifted to a lower wave vector on applying the electric field. 

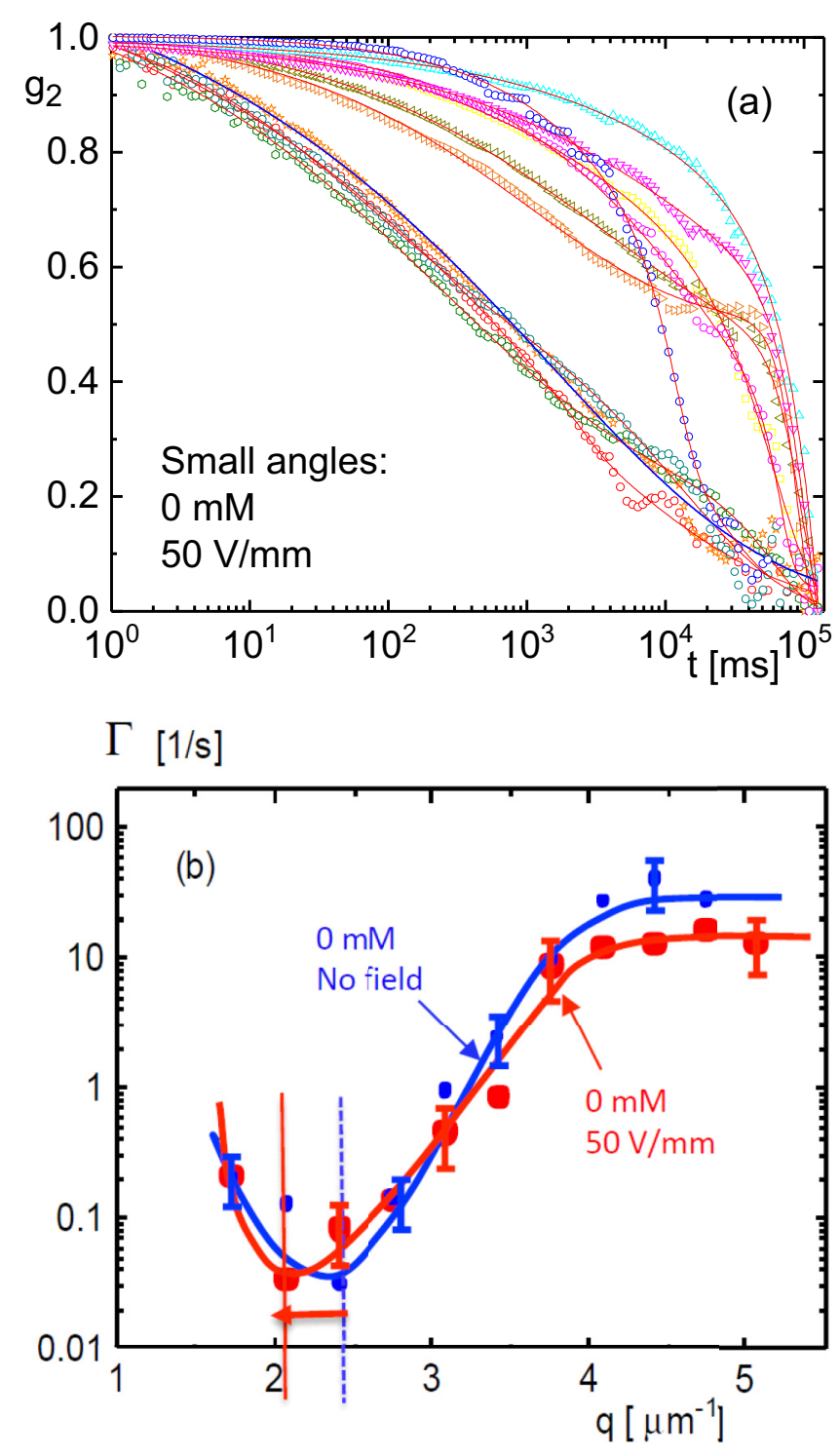

FIG. 4. (a) Small-wave vector correlation functions in the presence of an electric field with amplitude $50 \mathrm{~V} / \mathrm{mm}$ for the dispersion without added salt. The color code is the same as in Fig. 3(a). (b) The corresponding dispersion relation (the red data points) and the dispersion relation for the sample without added salt and without an electric field as determined from the correlation functions in Fig. 3(b) (the blue data points). The decay rates are now determined as the reciprocal time at which the normalized correlation functions decayed to a value of 0.7 . The solid lines in (b) are guides to the eye.

\section{B. The ionic-strength dependence of long-wavelength density fluctuations}

For CNC suspensions with a concentration of $5 \mathrm{wt} \%$, the chiral-nematic state is the stable state for concentrations of added salt up to about $5 \mathrm{mM}$ added $\mathrm{NaCl}$ (see the discussion in the Introduction and Fig. 1). Figure 5(a) shows the low$q$ correlation functions with $2 \mathrm{mM}$ added salt, without an electric field, and in Fig. 5(b) in the presence of an electric field with amplitude $25 \mathrm{~V} / \mathrm{mm}$. Figure 5(c) shows the corresponding dispersion relations, including that for $0 \mathrm{mM}$ without an external field (the black data points). Note that a decay value of 0.5 that has been used in Fig. 3 would lead to rather large errors in the decay rates for the $10 \mathrm{mM}$ salt sample as can be seen especially from Fig. 5(e), which is the reason for choosing this value as 0.7 . As can be seen, in the absence of an electric field, a higher ionic strength shifts the minimum to a smaller wave vector with a smaller decay rate, whereas the electric field shifts the minimum to significantly larger wave vectors. The former is most probably related to the diminishing extent of the repulsive electrostatic interactions, whereas the latter is most probably due to a combination of electric-field-induced inter-CNC interactions and alignment of the chiral-nematic domains. Since the effective concentration at the higher ionic strength is smaller, the chiral-nematic domains are much more easily aligned along the field direction as compared to the domains at zero added salt. The SAeDLS setup probes density fluctuations for wave vectors perpendicular to the direction of alignment so that the shift of the minimum in Fig. 5(c) on applying a field implies that the dynamics of density waves are strongly affected by orientations of the CNCs perpendicular to the direction of the density wave in the sense that the characteristic wavelength where the slow relaxation occurs is significantly shifted.

The same plots for CNC suspensions with $10 \mathrm{mM}$ added salt are shown in Figs. 5(d)-5(f). As discussed before, at this relatively high ionic strength, the dispersion is in a gel state without any chiral-nematic ordering. The dispersion relations in Fig. 5(f) are quite different from those in the chiral-nematic state. The clear minimum in the dispersion relation is not observed for the isotropic state. The functional form of the correlation functions in Figs. 5(d) and 5(e) for the isotropic sample is quite different from those in the nematic state. Contrary to the nematic samples, the isotropic sample exhibits two quite distinct relaxation modes. The decay rates determined from the inverse time at which the correlation functions decayed to a value of 0.7 clearly relate to the fast mode, and therefore, Fig. 5(f) also relates to the fast mode. As can be seen from Figs. 5(d) and 5(e), the decay rates of the slow mode are less than about $10^{-4} \mathrm{~s}^{-1}$, quite independent of the scattering vector. The dispersion relations in the isotropic phase and in the nematic phase are, thus, fundamentally different. The effect of the electric field is now to reduce the relaxation rates [see the red data points in Fig. 5(f)].

We observed an increase in the relaxation times of correlation functions for $20 \mathrm{mM}$ added salt during time as can be seen in Fig. 6(a), which is attributed to CNC aggregation, in agreement with an earlier study on the phase behavior of CNCs [6]. On applying an electric field, oscillating correlation functions are obtained [see Fig. 6(b)], which is attributed to field-induced migration of the aggregates. Aggregation, thus, precludes the determination of dispersion relations for this high salt concentration.

The trends found in our experiments for the saltconcentration and electric-field dependences of the location of the wave vectors $q_{c}$ and the corresponding critical length scale where the minimum in the dispersion relations is found are as follows. In the absence of an electric field, in the nematic state, there is a modest increase in the critical length $\xi_{c}=2 \pi / q_{c}$ (with $q_{c}$ as the wave vector where the minimum occurs) with increasing salt concentration [see Fig. 5(c)]. The effect of the electric field is quite minor without added salt [see 

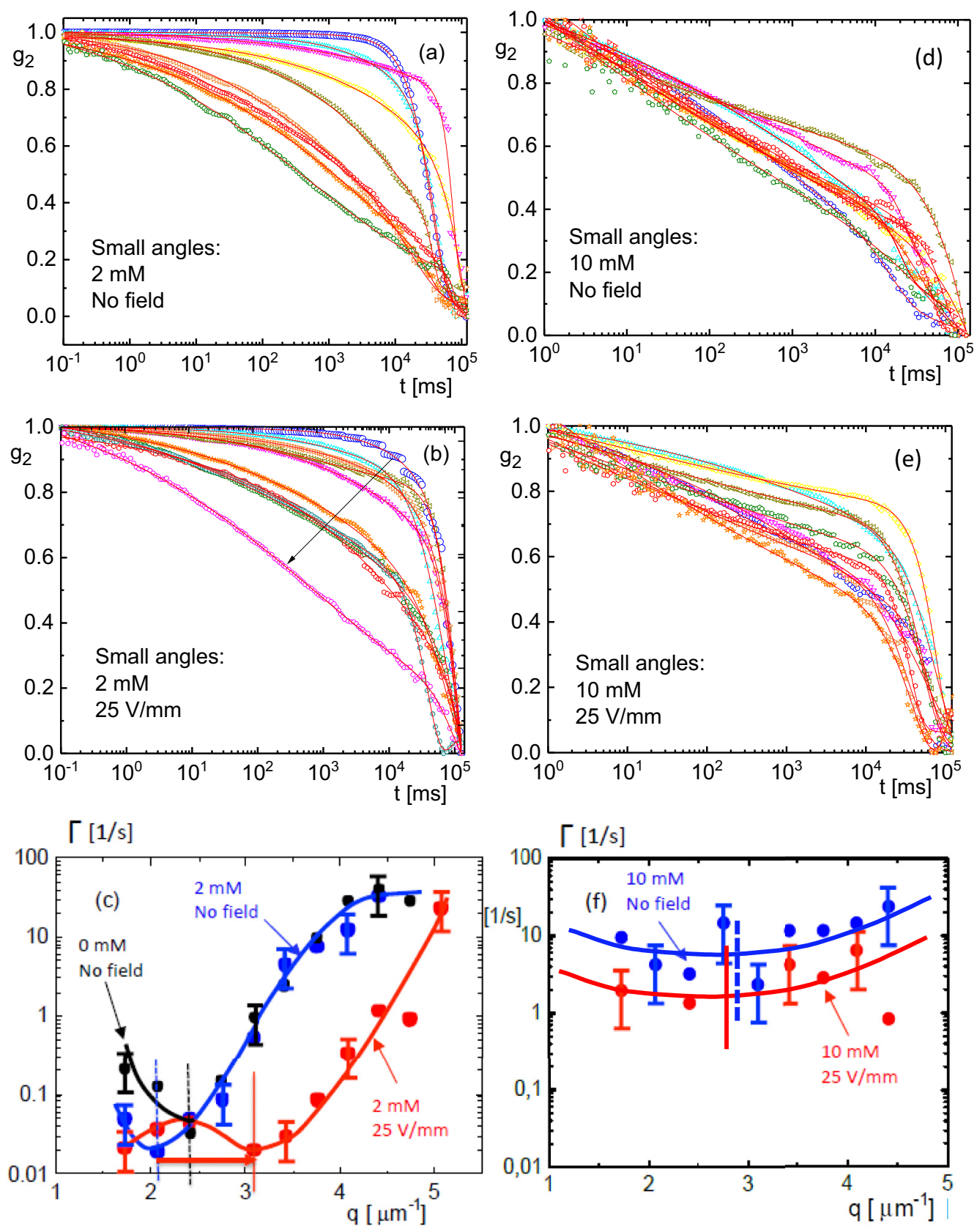

FIG. 5. Small-wave vector correlation functions for the CNC suspension with (a)-(c) $2 \mathrm{mM}$ and (d)-(f) $10 \mathrm{mM}$ added salt: (a) and (d) without and (b) and (d) with an electric field (the amplitude of which is $25 \mathrm{~V} / \mathrm{mm}$ ). The color codes are the same as in Fig. 3. (c) and (f) The dispersion relations without field (the blue points), with field (the red points), and in (c) for $0 \mathrm{mM}$ without field (the black points), which are taken from Fig. 4(b). The solid lines are a guide to the eye.

Fig. 4(b)] and quite pronounced for higher salt concentrations [see Fig. 5(c)] where the critical length shifts to lower values. The main effect of the electric field in the isotropic gel state is to decrease relaxation rates [see Fig. 5(f)].

\section{Temperature dependence of relaxation rates within the chiral-nematic phase}

So far, we have discussed density relaxation of CNCs at room temperature. In this subsection, preliminary results for the temperature dependence of relatively short-wavelength density fluctuations are discussed for the chiral-nematic state at a CNC concentration of $5 \mathrm{wt} \%$ and with $2 \mathrm{mM}$ added $\mathrm{NaCl}$. Measurements with the wide-angle DLS setup in the $\mathrm{VH}$ mode in the absence of an electric field are performed at 1,22 , and $32{ }^{\circ} \mathrm{C}$. Correlation functions for these temperatures, each at wave vectors $14.7,19.9$, and $24.8 \mu \mathrm{m}^{-1}$, are given in Figs. 8(a)-8(c). As discussed in Sec. III, the large-wave vector $\mathrm{VH}$-mode correlation functions exhibit three dynamical modes, which are attributed to fast translational diffusion along the chiral-nematic director, an intermediate translational mode related to motion perpendicular to the director, and a slow mode. The correlation functions are fitted to Eq. (4) for the field-correlation function together with the homodyne 


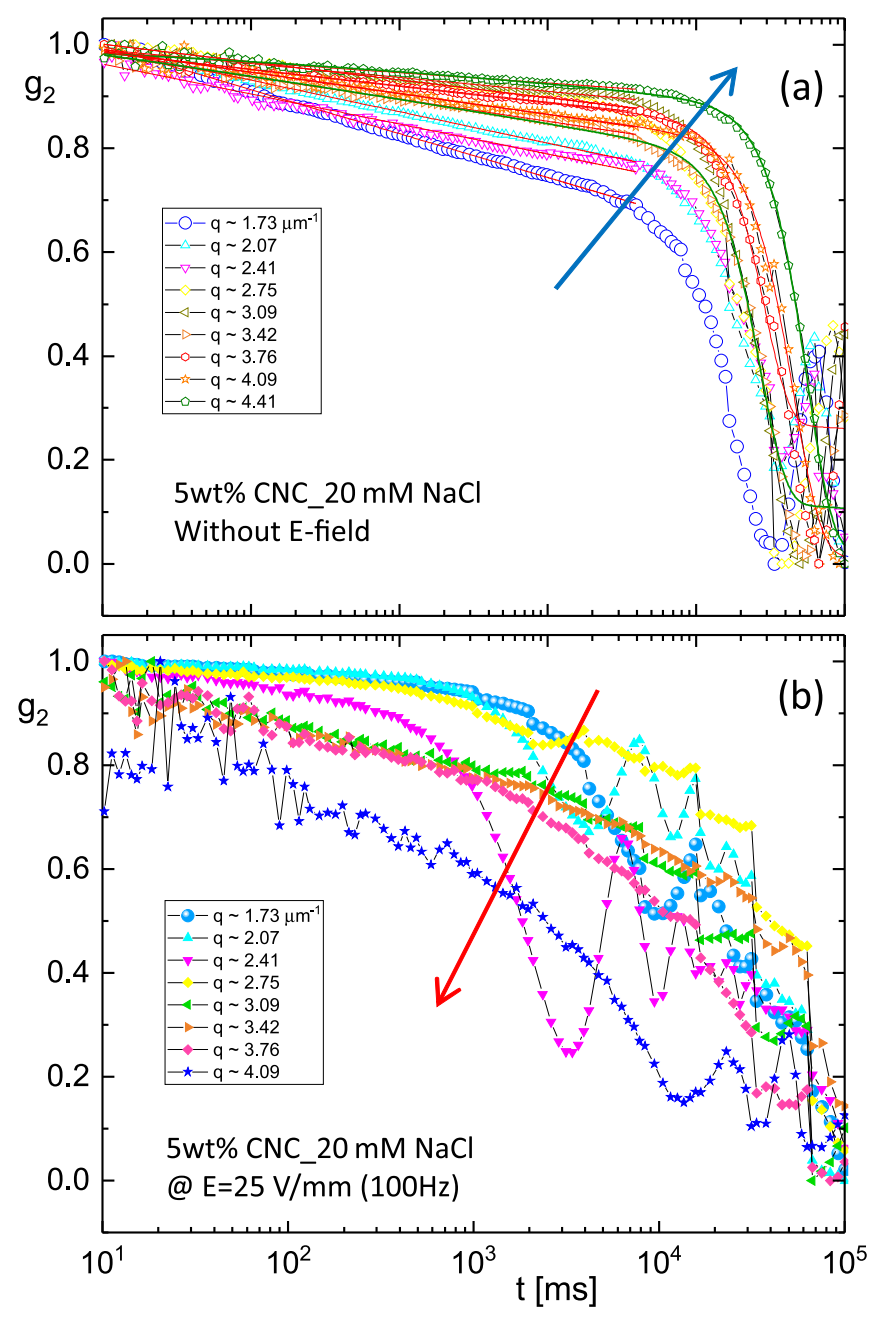

FIG. 6. The VH-correlation functions for $20 \mathrm{mM}$ added salt, (a) without field and (b) with the electric field with an amplitude of $25 \mathrm{~V} / \mathrm{mm}$. The color codes are the same as in Fig. 3. Field-induced oscillations are visible due to the possible drift velocity of $\mathrm{CNC}$ aggregates.

Siegert relation. The solid lines in Figs. 8(a)-8(c) are the fitting results. The amplitudes of the fast mode vary between 0.02 and 0.05 , the amplitudes of the intermediate mode vary from 0.05 to 0.09 , whereas the major relaxation is due to the slow mode with an amplitude on the order of 0.90 . Since the slow mode is dominant with respect to the other modes, the slow mode is, therefore, the mode that has been probed in previous sections. The relaxation rates as functions of the wave vector for the three temperatures as obtained from the fits to Eq. (4) are plotted in Figs. 7(d)-7(f) for the fast-, intermediate-, and slow-mode, respectively.

When structural order is independent of temperature, relaxation rates will monotonically increase with increasing temperature. Note that the viscosity of water decreases from $0.0013 \mathrm{~Pa} \mathrm{~s}$ at $12{ }^{\circ} \mathrm{C}$ to $0.0008 \mathrm{~Pa} \mathrm{~s}$ at $30^{\circ} \mathrm{C}$, which enhances the dynamics even more with increasing temperature as compared to simple fluids. The nonmonotonic temperature dependence of the relaxation rates seen in Figs. 7(d)-7(f) is, therefore, a signature for the temperature dependence of the microstructural order of the $\mathrm{CNCs}$ in the chiral-nematic state. To within experimental error [which is less than $10 \%$ for the relaxation rates in Figs. 7(d)-7(f)] such a monotonic behavior is not in contradiction to what is found for the slow and intermediate modes. In contrast, a nonmonotonic behavior is seen for the fast mode for the smaller wave vectors, which relaxation mode is attributed to motion of CNCs parallel to the chiral-nematic director. Figure 8 illustrates the possible collective particle alignments of CNCs at different temperatures. The pronounced increase in the relaxation rate with increasing temperature as found for the fast mode hints to an increasing degree of alignment.

The above observations validate the analysis of correlation functions in previous sections in terms of the relaxation rate related to the very slow mode (since the amplitude of this mode is dominant). The nontrivial temperature dependence of the dispersion relations should be investigated in more detail in the future, including extensive computer simulations on quite large systems of rodlike colloids in the chiral-nematic state in order to connect the dynamics to changes in the temperature-dependent microstructural ordering of these systems. These changes in microstructural order are determined by the inter-CNC interactions, which include electrostatic repulsion, van der Waals attraction, and excluded (chiral) core-core interactions.

\section{SUMMARY AND CONCLUSIONS}

Dynamic light scattering on CNC dispersion relations have been performed at small wave vectors in order to probe the relaxation of long-wavelength density fluctuations. In particular, the chiral-nematic state of CNCs is explored by depolarized light scattering (in the $\mathrm{VH}$ mode), which is sensitive to the $\mathrm{CNC}$-orientation dependence of the relaxation of density waves, contrary to polarized light scattering (in the $\mathrm{VV}$ mode). In the $\mathrm{VH}$ mode, three relaxation mechanisms can be distinguished: A fast relaxation due to motion of the $\mathrm{CNC}$ rods along their long axis, that is, along the director, an intermediate mode that is due to diffusion perpendicular to the director, and a very slow mode that is attributed to the coupling of translation to the relatively slow orientational relaxation of the CNCs. The contribution to the scatteredintensity correlation functions of the latter is much larger than the two former relaxation modes. An analysis of the slowest mode reveals a strong dependence of the relaxation rate of long-wavelength density fluctuations on the orientation of the rodlike CNCs as evidenced by nonmonotonic dispersion relations as obtained from scattering data in the $\mathrm{VH}$ mode. These dispersion relations exhibit multiple minima. The coupling of orientation to translational motion always leads to smaller relaxation rates as probed in the VV mode.

The occurrence of pronounced nonmonotonic dispersion relations is found to occur only in the chiral-nematic state and is essentially absent in the gel state for $10 \mathrm{mM}$ added salt. For a larger salt concentration of $20 \mathrm{mM}$ salt, the relaxation times increase during time due to aggregation, in accord with earlier determined phase diagrams of CNC dispersions.

At very low ionic strength (no added salt), the dispersion relation for the $\mathrm{VH}$ mode is relatively insensitive to the application of an external electric field. With $2 \mathrm{mM}$ added salt, on the contrary, there is a pronounced shift of the first 

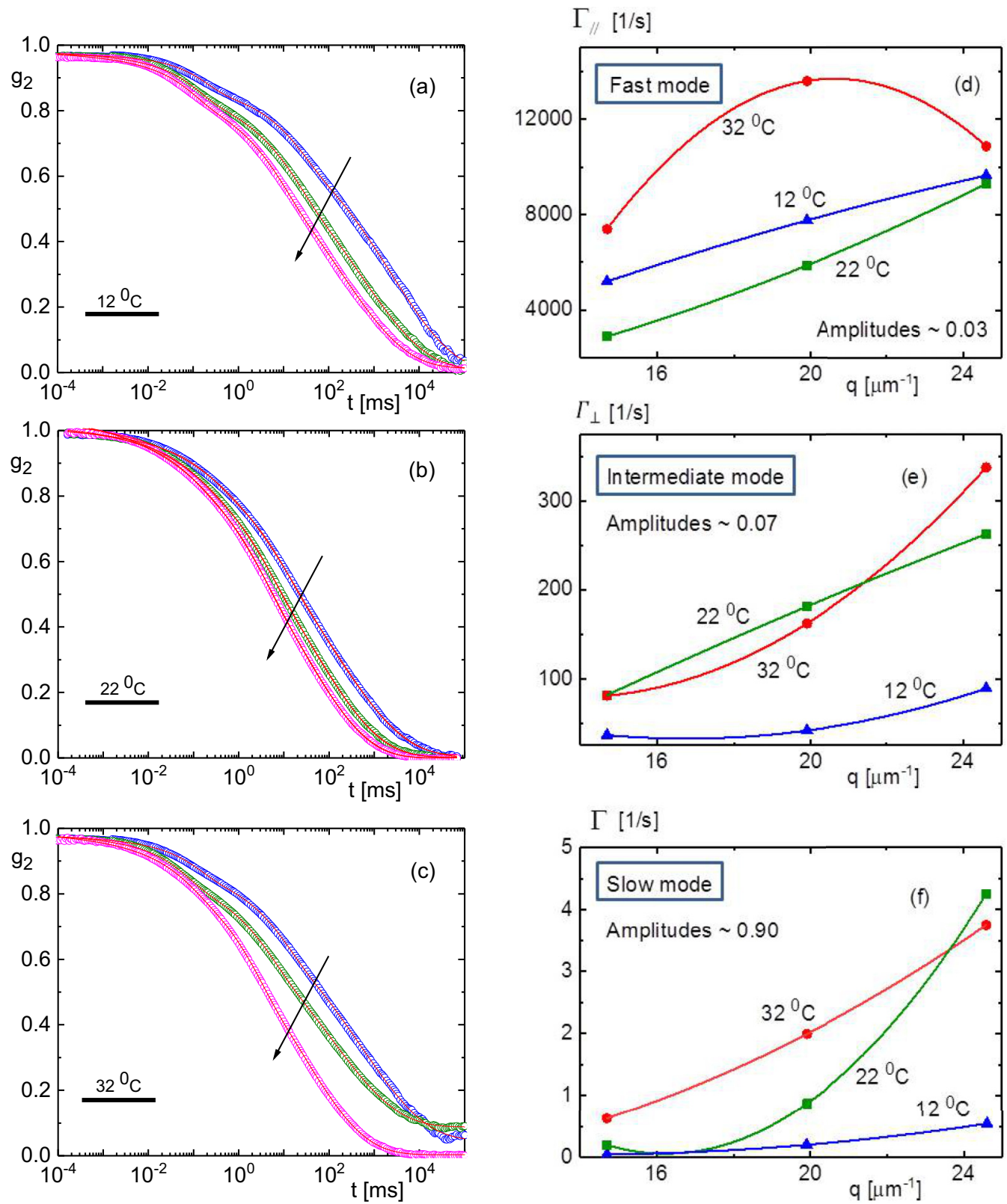

FIG. 7. Wide-angle depolarized (VH-) intensity autocorrelation functions in the absence of an electric field for a 5 wt $\%$ CNC suspension with $2 \mathrm{mM}$ added $\mathrm{NaCl}$ for three different temperatures: (a) $32^{\circ} \mathrm{C}$, (b) $22^{\circ} \mathrm{C}$, and (c) $12^{\circ} \mathrm{C}$. The wave vectors at which the correlation functions are measured are $14.7,19.9$, and $24.8 \mu \mathrm{m}^{-1}$. Note that, at a higher temperature (of $32^{\circ} \mathrm{C}$ ), a high but visibly saturated value of the relaxation rates of the fast mode (in the parallel component) are shown in (d) as compared to the increase in perpendicular relaxation [in (e)]. The brief interpretations of dispersion relations are sketched in Fig. 8.

minimum in the dispersion relation to larger wave vectors. A nontrivial temperature dependence of the dispersion relations at relatively large wave vectors is observed, which must be related to the change in the microstructural arrangement of the CNCs with temperature.

Contrary to thermotropic systems where the size of the molecules is much smaller than the colloidal CNCs under consideration here, the interpretation light-scattered correlation functions cannot be based on the well-known hydrodynamic theory for liquid-crystalline systems in terms of splay, twist, and bend fluctuations. The wavelength $2 \pi / q$ in the experimental dispersion relation in Fig. 3(d) varies from 45 to 3 times the length of a single $\mathrm{CNC}$, whereas macroscopic structural features extend over tens of microns as can be seen from Fig. 1, which is larger than the maximum wavelength of $3.7 \mu \mathrm{m}^{-1}$ corresponding to the smallest scattering angle. The interpretation of correlation functions on the basis of such a hydrodynamic theory is, therefore, questionable. In addition, dispersion relations based on such a hydrodynamic theory cannot explain the nonmonotonic behavior found in our experiments. The nonmonotonic behavior of the dispersion relation can only be explained on a molecular level.

The molecular origin of the above described behavior of long-wavelength fluctuations is related to the mutual CNC interactions, which are a combination of electrostatic repulsion, van der Waals interactions, and chiral core-core 


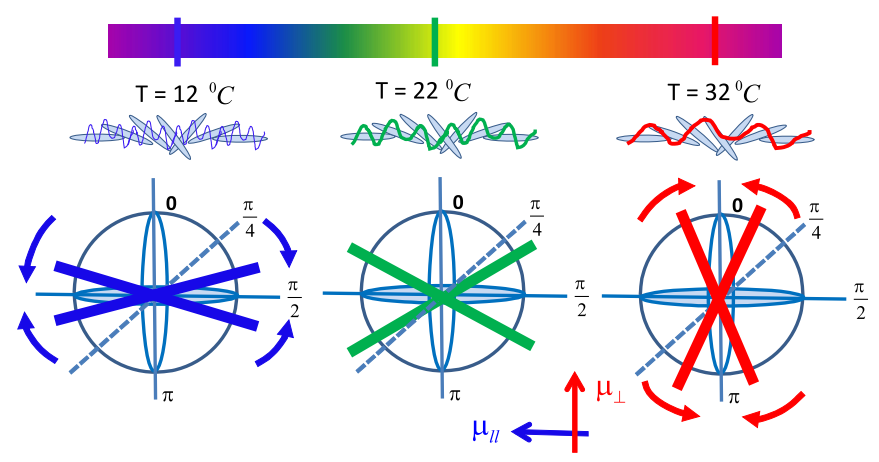

FIG. 8. Representation of the possible CNC motions giving rise to the observed relaxation modes for the three temperatures 12,22 , and $32^{\circ} \mathrm{C}$, corresponding to the anisotropic mobilities (indicated as $\mu_{\|}$and $\mu_{\perp}$ for parallel and perpendicular, respectively, to the chiral-nematic director). At lower temperature-driven fluctuations of CNCs, the parallel motions contribute more than the other two modes. However, at higher temperatures, thermal fluctuations of CNCs lead to a relatively saturated relaxation rate for the fast mode as compared to the other two modes, thus, resulting in increased perpendicular mobilities [see Fig. 7(e)].

interactions. A quantitative understanding of the molecular origin of long-wavelength fluctuations is beyond the scope of this paper and probably requires extensive simulations on quite large systems of chiral molecules. The observed strong dependence of density relaxation rates on the orientation of CNCs and the dependence of relaxation rates on an external electric field is probably more valid for any system of rodlike colloids in the chiral-nematic state and may have implications in processing inhomogeneous rodlike colloidal dispersions. The findings presented here may initiate efforts to obtain a microscopic understanding of origin of the nonmonotonic dispersion relations as well as the effect of ionic strength and externally applied electric fields for rodlike systems, in general, and CNC dispersions, in particular.

Finally, we hope that this paper will inspire further research on the orientation dependence of the dynamics of CNCs and (chiral-)nematics in the small wave vector regime, including the influence of electric fields and temperature. We hope this paper is useful to understand such anisotropic dynamics and to engineer other types of CNC suspensions for tuning collective orientations of particles.

\section{ACKNOWLEDGMENTS}

K.K. thanks J. K. G. Dhont for his scientific interests, and P.B. and P.F. acknowledge the Swiss National Science Foundation (Grant No. 200021-175994). We also acknowledge CelluForce for providing the CNCs.
[1] T. Li, J. Song, X. Zhao, Z. Yang et al., Sci. Adv. 4, eaar3724 (2018).

[2] P. Bertsch, A. Sánchez-Ferrer, M. Bagnani, S. Isabettini, J. Kohlbrecher, R. Mezzenga, and P. Fischer, Langmuir 35, 4117 (2019).

[3] P. Bertsch, S. Isabettini, and P. Fischer, Biomacromolecuels 18, 4060 (2017).

[4] Y. Xu, A. D. Atrens, and J. R. Stokes, Soft Matter 14, 1953 (2018).

[5] X. M. Dong, T. Kimura, J.-F. Revol, and D. G. Gray, Langmuir 12, 2076 (1996).

[6] P. Bertsch, M. Diener, J. Adamcik, N. Scheuble, T. Geue, R. Mezzenga, and P. Fischer, Langmuir 34, 15195 (2018).

[7] K. Kang and J. K. G. Dhont, Soft Matter 6, 273 (2010).

[8] M. Hagenbuechle, P. Sheaffer, Y. Zhu, and J. Liu, Int. J. Mod. Phys. B 10, 3057 (1996).
[9] M. Hagenbuechle and J. Liu, Int. J. Mod. Phys. B 13, 2077 (1999).

[10] Y. Xu, A. D. Atrens, and J. R. Stokes, J. Colloid Interface Sci. 496, 130 (2017).

[11] A. Kadimi, K. Benhamou, Z. Ounaies, A. Magnin, A. Durresne, H. Kaddami, and M. Raihane, Appl. Mater. Interfaces 6, 9418 (2014).

[12] S. Shafrei-Sabet, W. Y. Hamad, and S. G. Hatzikiriakos, Langmuir 28, 17124 (2012).

[13] Y. Xu, A. D. Atrens, and J. R. Stokes, Soft Matter 15, 1716 (2019).

[14] K. Kang, Rev. Sci. Instrum. 82, 053903 (2011).

[15] J. K. G. Dhont, An Introduction to Dynamics of Colloids (Elsevier, Amsterdam, 1996).

[16] C. Schuetz, J.van Rie, S. Eyley, A. Gencer et al., Sustainable Chem. Eng. 6, 8317 (2018). 